\title{
Photophysical Properties of Blue-Emitting Silicon Nanoparticles
}

\author{
Manuel J. Llansola Portolés, ${ }^{\dagger}$ Felipe Rodriguez Nieto ${ }^{\dagger}{ }^{\dagger}$ Delia B. Soria, ${ }^{\ddagger}$ Javier I. Amalvy, ${ }^{\dagger}$ \\ Pablo J. Peruzzo, ${ }^{\dagger}$ Daniel O. Mártire, ${ }^{\dagger}$ Mónica Kotler, ${ }^{\S}$ Oliver Holub,,${ }^{\prime \prime}$ and \\ Mónica C. Gonzalez ${ }^{*, \dagger}$ \\ Instituto de Investigaciones Físicoquímicas Teóricas y Aplicadas (INIFTA), Facultad de Ciencias Exactas, \\ Universidad Nacional de la Plata, c.c. 16, suc. 4, La Plata, Argentina, Departamento de Química, Biológica, \\ FCEyN, Universidad de Buenos Aires, 4 Piso, Pabellón II, Buenos Aires, Argentina, and Laboratory for \\ Fluorescence Dynamics, Department of Biomedical Engineering, 3206 Natural Sciences II Building, University \\ of California, Irvine (UCI), Irvine, California 92697-2715
}

Received: April 22, 2009; Revised Manuscript Received: June 11, 2009

\begin{abstract}
Silicon nanoparticles with strong blue photoluminescence were synthesized by electrochemical etching of silicon wafers and ultrasonically removed under $\mathrm{N}_{2}$ atmosphere in organic solvents to produce colloids. Thermal treatment leads to the formation of colloidal Si particles of $3 \pm 1 \mathrm{~nm}$ diameter, which upon excitation with 340-380 nm light exhibited room temperature luminescence in the range from 400 to $500 \mathrm{~nm}$. The emission and the one- and two-photon excitation spectra of the particles are not sensitive to surface functionalization with methyl 2-methylprop-2-enoate. However, the derivatized particles show higher emission quantum yields in air-saturated suspensions (44\%) than the underivatized particles (27\%), as well as higher stability of its dispersions. FTIR and XPS spectra indicate a significant surface oxidation of the particles. The Si:O:C ratio at the surface of the derivatized particles estimated from XPS is $\mathrm{Si}_{3} \mathrm{O}_{6}\left(\mathrm{C}_{5} \mathrm{O}_{2} \mathrm{H}_{y}\right)_{1}$, with $y=7-8$. Vibronic spacing is observed in both the emission and excitation spectra. The information obtained from one-photon excitation experiments (emission and excitation spectra, photoluminescence quantum yields, luminescence decay lifetimes, and anisotropy correlation lifetimes), as well as from two-photon excitation fluorescence correlation spectroscopy (brightness and diffusion coefficients) and TEM, indicate that the blue-emitting particles are monodisperse and ball-shaped. Particle size clearly determines the emission and excitation spectral region, as expected from quantum confinement, but the presence and extent of $\mathrm{Si}-\mathrm{O}$ species on the silicon networks seem crucial for determining the spectrum features and intensity of emission. The nanoparticles could hold great potential as quantum dots for applications as luminescence sensors in biology and environmental science.
\end{abstract}

\section{Introduction}

Silicon nanoparticles (Si-NPs) of $1-5 \mathrm{~nm}$ size received great attention, as they combine size-dependent photoluminescence (PL) with the richness of silicon surface chemistry. The momentum requirements that make bulk $\mathrm{Si}$ a rather inefficient light emitter are relaxed in the $1-5 \mathrm{~nm}$ diameter Si crystals as a result of quantum confinement effects. ${ }^{1}$ The PL of $\mathrm{H}-$ terminated nanocrystals may be tuned from the near-IR to the blue with decreasing particle size. ${ }^{2-4}$

The controlled synthesis of monodisperse batches of these nanoparticles (NPs) is of interest as materials for light-emitting structures for a variety of biochemical and engineering applications. ${ }^{5,6} \mathrm{Si}-\mathrm{NPs}$ have been prepared by several routes, including electrochemical HF-mediated etching of crystalline $\mathrm{Si}$ wafers to yield porous silica (PS) and further dispersion of the particles by ultrasound, ${ }^{3,4,7-9}$ chemical reaction of $\mathrm{Si}$ precursors in solution, ${ }^{1,10}$ and gas phase synthesis. ${ }^{11,12}$ However, significant differences are reported in the room temperature PL

* To whom correspondence should be addressed. E-mail: mcgonzalez.quim@gmail.com. Phone: +54-221-4257430. Fax: +54-2214254642 .

†NIFTA, Universidad Nacional de La Plata.

CEQUINOR, FCE, Universidad Nacional de La Plata.

§ Departamento de Química Biológica, FCEyN, Universidad de Buenos Aires, Argentina.

"University of California, Irvine (UCI). of apparently similar particles obtained by different synthetic routes, as discussed below.

Hydrogen-capped and methyl ester functionalized Si particles of $\sim 1 \mathrm{~nm}$ diameter prepared from anodically etched $\mathrm{PS}^{8}$ show a PL spectrum with maxima around 320 or $380 \mathrm{~nm}$ at excitation wavelengths of 270 and $320 \mathrm{~nm}$, respectively. Air-stable octanolcapped $1.6 \mathrm{~nm}$ mean diameter Si-NPs synthesized from a mixture of argon/silane in a continuous flow atmosphericpressure microdischarge reactor, were reported to exhibit PL that peaks at $420 \mathrm{~nm}$ and an excitation peak at $360 \mathrm{~nm} .{ }^{12} \mathrm{On}$ the other hand, alkyl-functionalized Si-NPs of $(1.6 \pm 0.2) \mathrm{nm}$ size synthesized by a solution reduction method in the presence of micelles, showed UV/vis absorption and emission spectra $\left(\lambda_{\mathrm{em}}{ }^{\max } \sim 280-290 \mathrm{~nm}\right)$ with vibrational progressions (974 \pm $14 \mathrm{~cm}^{-1}$ ) which resembled bulk SiC phonons. ${ }^{1}$ Octyl -capped Si-NPs of $(3.9 \pm 1.3) \mathrm{nm}$ diameter, synthesized by the metathesis reaction of sodium silicide with $\mathrm{NH}_{4} \mathrm{Br}$, exhibited PL with $\lambda_{\mathrm{em}}{ }^{\max } \sim 438 \mathrm{~nm}$ upon excitation at $360-400 \mathrm{~nm} .{ }^{13}$

In small crystalline - Si-NPs of sizes comparable to or smaller than the exciton Bohr radius in bulk $\mathrm{Si}(\sim 4.5 \mathrm{~nm}),{ }^{14,15}$ the wave functions of excitons and electrons are delocalized over the nanoparticles. Therefore, the optical properties of crystalline -Si nanoparticles and nanostructures are also sensitive to the surface chemistry and to the geometry of the structures. ${ }^{15}$ The important role of surface reconstruction and termination in determining the nature of the $1 \mathrm{~nm}$ - particles PL is well described by theoretical studies, which consider molecular-like energy 
levels. ${ }^{3,16,17}$ Studies performed for Si-NPs of 1- to $1.5-\mathrm{nm}$ size ${ }^{17}$ demonstrated that oxygen has a direct interaction with the $\mathrm{Si}$ orbitals that substantially changes the HOMO and LUMO with respect to those of $\mathrm{H}$-terminated particles. Thus, the emission of H-terminated nanocrystals above $3.0 \mathrm{eV}$ in the blue (emission peak with $\lambda_{\mathrm{em}}{ }^{\max }<400 \mathrm{~nm}$ ) moves to near $2.0 \mathrm{eV}$ in the yellowred $\left(\lambda_{\mathrm{em}}{ }^{\max } \sim 600 \mathrm{~nm}\right)$ upon oxidation. For these sizes, the excited state is a surface excitation directly involving the capping atoms rather than the volume excitation. The direction of the oxidation effect is opposite to that predicted by a quantum size model, which considers that only the number of elemental $\mathrm{Si}$ atoms in the nanocrystal core is responsible for the PL, as surface oxidation decreases the number of these $\mathrm{Si}$ atoms. In fact, studies reporting the preparation of oxidized stable blueemitting Si-NPs $\left(\lambda_{\mathrm{em}} \sim 460 \mathrm{~nm}\right)$ from the oxidation of $5 \mathrm{~nm}$ size red-emitting $\left(\lambda_{\mathrm{em}}{ }^{\max } \sim 600 \mathrm{~nm}\right)$ Si-NPs with an organic monolayer grafted to their surface support the quantum size model. ${ }^{11}$ The presence of oxygen is an evident reason for the observed discrepancies; still, the direction and magnitude of these differences is not clear.

With the aim of obtaining organically capped Si-NPs dispersible in most common solvents and with strong and stable emission for their use as luminescent markers in biological and environmental systems, we undertook the electrochemical etching of crystalline $\mathrm{Si}$ wafers and further dispersion of particles by ultrasound as a synthetic route. An adaptation of the method allowed us to obtain stable suspensions of functionalized SiNPs with emission lifetimes and spectra different from those previously reported for particles of similar size. Here, we report their synthesis and further derivatization with methanol and methyl 2-methylprop-2-enoate, as well as their characterization by transmission electron microscopy (TEM), Fourier transformed infrared spectroscopy (FTIR), X-ray photoelectron spectroscopy (XPS), luminescence lifetime, fluorescence correlation spectroscopy (FCS), and time-resolved anisotropy measurements, to show that the extent of surface oxidation is as important as particle size in determining the particle luminescence characteristics. Moreover, to evaluate their potential use in biological experiments, their two-photon excitation spectrum and absorption cross section were determined.

\section{Experimental Methods}

All chemicals used were purchased from Sigma-Aldrich and employed without further purification unless otherwise indicated. Toluene ( $\left(0.005 \% \mathrm{H}_{2} \mathrm{O}\right.$ content $\left.), 99.7 \%(\mathrm{GC})\right)$, methanol, and methyl 2-methylprop-2-enoate (MMA) were deaerated before reaction. When required, toluene and methanol were dried over molecular sieves for $24 \mathrm{~h}$.

For the synthesis of Si-NPs, a modification of the electrochemical etching of porous Si published in the literature was employed. ${ }^{3,8,18,19}$ Clean crystalline Si p-type 100 wafers with resistivities between 1.0 and $10.0 \Omega \mathrm{cm}$ were electrochemically etched in a Teflon cell containing an electrolytic solution (3\% $\mathrm{HF}, 22 \% \mathrm{CH}_{3} \mathrm{OH}$, and $\left.25 \% \mathrm{HNO}_{3}(\mathrm{v} / \mathrm{v})\right)$. A $5-20 \mathrm{~mA} / \mathrm{cm}^{2}$ galvanostatic current was applied between the anode ( $\mathrm{Si}$ wafer) and the cathode (Pt electrode). The silicon wafer was immersed in the electrochemical bath at a constant rate of $1 \mathrm{~mm} / \mathrm{h}$ for 20 h. After etching, the wafer was rinsed with methanol, dried under nitrogen, and further immersed in deaerated toluene. Approximately $80 \mu \mathrm{g}$ of Si-NPs were released by ultrasound (Ney Dental Inc.; 50/60 Hz; $100 \mathrm{~W}$ ). Pulverization of the SiNP colloids in different organic solvents was also investigated. Under identical experimental conditions, toluene yields very stable suspensions (vide infra) with the highest PL. Therefore, toluene was mainly used as the solvent for further surface derivatization.

The derivatization of the particles was performed with batches of "freshly" prepared Si-NPs suspended in toluene in the absence of oxygen. Methyl coating is obtained after $4 \mathrm{~h}$ treatment of the toluene suspensions with $40 \mathrm{mM}$ methanol at $90{ }^{\circ} \mathrm{C}$. Coating with MMA is obtained after $4 \mathrm{~h}$ irradiation of the Si-NP suspensions containing 10 mM MMA with a Rayonet Photochemical Reactor Lamp (RPR 3500 A, Southerm N.E. Ultraviolet Co.) emitting at $350 \pm 20 \mathrm{~nm}$. A similar methodology for the coating with hydrocarbons is reported in the literature. ${ }^{20,21}$

The resulting Si-NP suspension was passed through hydrophilic $0.1 \mu \mathrm{m}$ PVDF Durapore VVPP Membrane Filters (Millipore) to eliminate solid residues, the solvent evaporated, and the particles further dispersed in other solvents of interest. Solvent evaporation and resuspension of the derivatized particles does not change the characteristics of their emission and excitation spectra. Contrary to this observation, underivatized particles showed a spectrum expanded to the red after solvent evaporation to dryness and resuspension, probably due to particle-particle interactions, leading to an irreversible agglomeration, as dilution does not appreciably change the observed spectrum.

FTIR spectra were obtained with a Bruker EQUINOX spectrometer. Either $\mathrm{KBr}$ disks or silicon wafers were used as holders. Disks were prepared by mixing vacuum-dried Si-NP toluene suspensions with dried $\mathrm{KBr}$ and pressed into a pellet. Otherwise, a small piece of Si wafer was dip-coated with a toluene suspension of $\mathrm{Si}-\mathrm{NPs}$ and dried at room temperature. Spectra were taken in the $4000-400 \mathrm{~cm}^{-1}$ range with $4 \mathrm{~cm}^{-1}$ resolution and corrected for the background signal by collecting 64 scans for each measurement. Attenuated total reflection infrared (ATR-IR) spectroscopy used for the analysis of the surface of the nanoparticles was obtained with a NICOLET 380 FTIR ATR accessory using a ZnSe crystal and an incident beam angle of $45^{\circ}$. Sixty-four scans at $4 \mathrm{~cm}^{-1}$ resolution were collected.

The XPS spectra were obtained under UHV with an XR50 Specs $\mathrm{GmbH}$ spectrometer with $\mathrm{Mg} \mathrm{K}(\alpha)$ as the excitation source and a PHOIBOS 100 half sphere energy analyzer. Calibration was performed with $\mathrm{Au} 4 \mathrm{f} 7 / 2$ and $\mathrm{Cu} 2 \mathrm{p} \mathrm{3/2}$ with binding energies of 84.00 and $933.67 \mathrm{eV}$, respectively.

TEM samples were prepared by dipping a carbon-coated 300mesh copper grid into a Si-NP suspension in toluene and the solvent evaporated in air. TEM micrographs were taken with a Phillips CM-10 microscope operating at $100 \mathrm{kV}$ at five different spots of each grid.

Optical measurements were performed at atmospheric pressure and at room temperature.

Electronic absorption spectra were recorded in a quartz cuvette $(1 \mathrm{~cm}$, Hellma) with a Cary $13 \mathrm{UV} / \mathrm{vis}$ double-beam spectrophotometer (Varian, Australia).

Steady-state luminescent measurements were performed with a Perkin-Elmer LS-50B spectrometer equipped with a Xe lamp as the excitation source, a monochromator for selecting the excitation wavelength with a bandpass gap of $3 \mathrm{~nm}$, and a sensitive-to-the-red R928 PMP detector. All spectra were corrected for the wavelength-dependent sensitivity of the detector and the source by recording reference data simultaneously. Additionally, emission spectra were corrected for Raman scattering by using the solvent emission spectrum.

To estimate the quantum yield of the synthesized Si nanoparticles, PL emission spectra were collected at various excita- 
tion wavelengths. Identical measurements (excitation conditions, lamp energy, and spectrometer bandpass) were performed with 9,10-diphenylanthracene in cyclohexane, which emits between 400 and $500 \mathrm{~nm}$ with a known efficiency of $90 \%$ upon $275-405$ $\mathrm{nm}$ excitation. ${ }^{22}$ The absorbance of argon-saturated toluene suspensions of the NPs at the excitation wavelength was used for the quantum yield calculations to avoid introducing a further source of error due to the charge transfer absorption of toluene-oxygen mixtures. ${ }^{23}$

Frequency-domain luminescence and anisotropy measurements were performed with a multifrequency cross-correlation phase and modulation fluorometer based on ISS Koala automated sample compartment, modulatable LEDs (280, 295, 370, 405, 471, 443, and $630 \mathrm{~nm}$ ), and a modulatable Hamamatsu photomultiplier (up to $500 \mathrm{MHz}$ ). A pulsed light-emitting diode $\left(\lambda_{\text {exc }}=405 \mathrm{~nm}\right)$ was used as the excitation source. Excitation light was passed through a $405 \pm 10 \mathrm{~nm}$ filter. Polarizers were used in a magic-angle position $\left(54.7^{\circ}\right)$ when no anisotropy was measured.

Two-photon scanning fluorescence correlation spectroscopy (FCS) measurements were performed with an Olympus IX70 microscope equipped with a UPlus Sapo Olympus $60 \times, 1.20$ V objective coupled with a Coherent Chameleon-Ultra femtosecond Ti-sapphire near-infrared laser, a Cambridge Technologies mirror scanner, and a Hamamatsu H7422P-40 PMT. The laser generated pulses of $150 \mathrm{fs}$ duration at a repetition rate of $80 \mathrm{MHz}$ and $2.53 \mathrm{~W}$ at $774 \mathrm{~nm}$. The excitation light was limited by two Schott RG715 filters to cut off light of $\lambda<715 \mathrm{~nm}$. Three filters, a HQ 520/90M, a dichroic 435/70 M, and a 470/ $30 \mathrm{M}$, limited the detected emission light to the $440-500 \mathrm{~nm}$ range, detected in the photon counting mode. Data were taken with a $128 \mathrm{kHz}$ frequency and a time base of $7.81 \times 10^{-6} \mathrm{~s}$, to obtain 1023 cycles in a total time of $261.888 \mathrm{~s}$ and using 512 bins.

A $1-2 \mathrm{~mm}$ radio Teflon ring was used as a cell to avoid evaporation effects. The excitation volume waist of the setup is determined from the autocorrelation function of rhodamine 110 , taken under the same beam focusing conditions. Assuming a diffusion coefficient for rhodamine of $D=3 \times 10^{-6} \mathrm{~cm}^{2} / \mathrm{s},{ }^{24}$ the laser is calculated to be focused to a spot of $0.37 \mu \mathrm{m}$ diameter. SimFCS and Globals, UCI developed programs, were used for analyzing the signals.

The signal-to-background ratio defined as the photon count rates of the excited molecules in the focused volume divided by the photon count rates of the background (e.g., solvent) is $\sim 20$ for the rhodamine experiments and $\sim 34$ for the nanoparticle experiments, under identical conditions.

Bilinear Regression Analysis. For each excitation wavelength of the particle suspension, an emission spectrum is obtained. For a pure substance existing in a unique form, the luminescence spectrum is invariant, remaining the same independent of the excitation wavelength. For low fluorophore absorbance $(<0.05)$, the steady-state emission intensity at a given emission wavelength $\left(I\left(\lambda_{m}\right)\right)$ is proportional to the absorption coefficient of the fluorophore at a given excitation wavelength $\left(\varepsilon\left(\lambda_{x}\right)\right)$, the concentration of the fluorophore, and the factor $F\left(\lambda_{m}\right)$ reflecting the distribution of the probability of the various transitions from the lowest vibrational level of the first electronic excited level to the various vibrational levels of the ground state. ${ }^{25}$ A bilinear regression analysis taking advantage of the linearity of $I\left(\lambda_{m}\right)$ with both $\varepsilon\left(\lambda_{x}\right)$ and $F\left(\lambda_{m}\right)$ was applied to the experimental emission matrix in order to retrieve information on the minimum number of species and on their relative emission and absorption spectra. ${ }^{26}$ Using the running indexes $x$

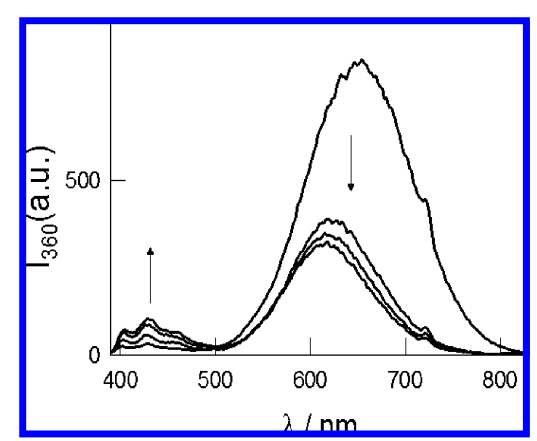

Figure 1. Time evolution of the emission spectra obtained at $360 \mathrm{~nm}$ excitation of as-obtained Si-NPs in toluene suspension. The arrows indicate the direction of the increasing and decreasing emission after $2,5,16$, and 25 days.

and $m$ for the excitation and emission wavelengths, respectively, in a matrix form $\left[i_{x m}\right]=\left[\varepsilon_{x j}\right] \cdot\left[\mathrm{f}_{j m}\right]$, where $\left[i_{x m}\right]$, $\left[\varepsilon_{x j}\right]$, and $\left[\mathrm{f}_{j m}\right]$ represent the emission intensity, absorption coefficients, and $F\left(\lambda_{m}\right)$ matrices, respectively. The procedure is based on the alternating calculus of the matrices $\left[\varepsilon_{x j}\right]$ and $\left[f_{j m}\right]$ by multiple linear regression iteratively until convergence is achieved when a least-squares condition is applied in order to minimize the elements of an error matrix. From the error matrix, a corrected emission intensity matrix, $\left[\mathrm{i}_{x m}{ }^{\mathrm{c}}\right]$, is obtained. The number of species is selected as the minimum that shows a reasonable approximation between $\left[\mathrm{i}_{x m}{ }^{\mathrm{c}}\right]$ and $\left[\mathrm{i}_{x m}\right]$.

\section{Results and Discussion}

"As-Obtained" Si-NPs. The excitation and emission spectra of the "as-obtained" particles in toluene strongly depend on aging. The suspension was sonicated for $30 \mathrm{~min}$ before measurement to avoid the slow precipitation of reddish luminescent particles. Figure 1 shows the evolution of the emission spectrum obtained by exciting a toluene suspension of the particles with $360 \mathrm{~nm}$ light at different times after the synthesis. The broadband with a maximum at $650 \mathrm{~nm}$ observed $2 \mathrm{~h}$ after the synthesis is shifted to the blue and decreased in intensity with aging, while a new emission band with a maximum around $440 \mathrm{~nm}$ is observed. Si-NPs obtained from pulverization of PS in methanol show a similar behavior, though lower luminescence intensities are observed.

The FTIR spectra of as-obtained Si-NPs supported on $\mathrm{KBr}$ disks show very weak and broad absorption peaks at 2100-2250 and $2200-2300 \mathrm{~cm}^{-1}$ (see Figure $2 \mathrm{~A}$, inset) which could be due to $\mathrm{Si}-\mathrm{H}_{x}$ stretching modes and $\mathrm{O}_{x} \mathrm{Si}-\mathrm{H}$ vibrations, respectively. Accordingly, the peak at $\sim 900 \mathrm{~cm}^{-1}$ could be assigned to $\mathrm{Si}-\mathrm{H}$ bending modes. These results are in agreement with those usually observed for planar hydrosilylated Si surfaces. ${ }^{27-31}$ The additional bands observed at nearly 3400, 1630, and 800 $\mathrm{cm}^{-1}$ are assigned to $\mathrm{Si}-\mathrm{OH}$ groups and those at $1100 \mathrm{~cm}^{-1}$ to $\mathrm{Si}-\mathrm{O}-\mathrm{Si}$ vibrations which characterize the spectrum of silica nanoparticles $\left(\mathrm{SiO}_{2}\right.$-NPs). ${ }^{28,29,32}$

Four hours of heating at $90{ }^{\circ} \mathrm{C}$ of toluene suspensions of freshly prepared Si-NPs under nitrogen has almost the same effect on the luminescence evolution of the particles than 2 weeks of aging at room temperature. Therefore, this procedure was applied to optimize the synthesis of the blue-emitting particles. However, in order to avoid surface oxidation of the particles during workout, derivatization was performed with batches of freshly prepared Si-NPs suspended in toluene in the absence of oxygen, without previous thermal treatment. Filtration of suspensions of either underivatized particles or methanol or MMA derivatized particles with $0.1 \mu \mathrm{m}$ PVDF filters or 10 


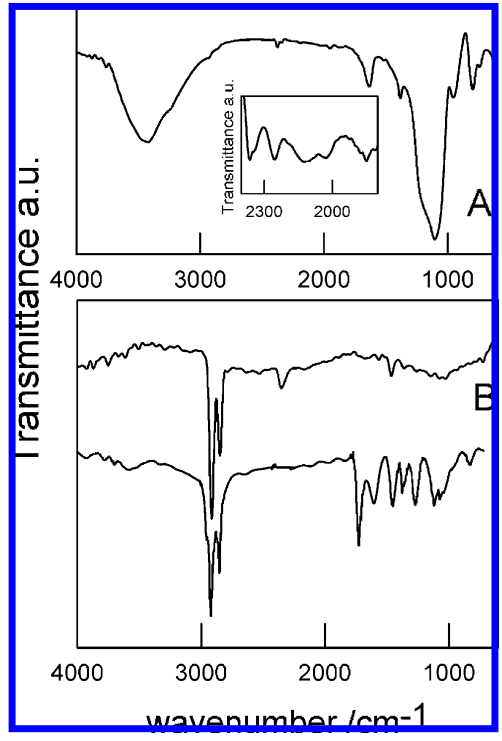

Figure 2. FTIR spectrum of (A) HOSi-NPB supported on a silicon wafer. Inset A: "As-obtained" silicon particles supported on $\mathrm{KBr}$ pellets. (B) Top spectrum: MeSi-NPB supported on Si wafer. Bottom spectrum: ATR-IR spectrum of MMASi-NPB supported on Si wafer.

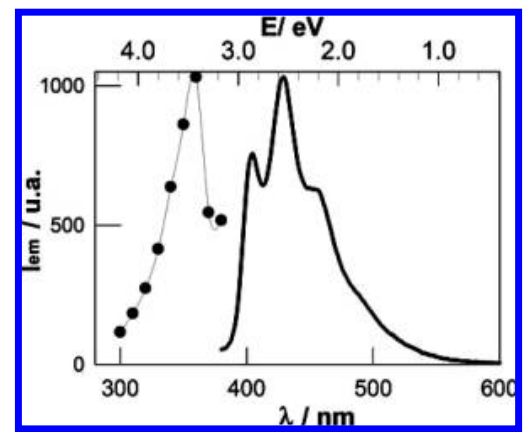

Figure 3. Emission and excitation spectra of HOSi-NPB suspensions in toluene retrieved from the bilinear regression analysis of the excitation-emission matrix. The emission spectrum was normalized to the excitation at $360 \mathrm{~nm}$ and the excitation spectrum to the emission at $425 \mathrm{~nm}$.

min centrifugation with a force of $10062 g$ allows the mechanical separation of the blue-emitting particles which remain in suspension from those emitting in the red, retained in the filter.

Blue-Emitting Particles. Underivatized Blue-Emitting Particles: HOSi-NPB . A bilinear analysis ${ }^{26}$ of the emission intensity matrix of the filtered suspension indicates the existence of one well-defined population of particles emitting in the blue, as the emission spectrum does not depend on the excitation wavelength. The excitation and emission spectra are shown in Figure 3 . The FTIR spectrum of these particles, from now on denoted as HOSi-NPB, shows peaks due to $\mathrm{Si}-\mathrm{OH}(3400-3500,1630$, and $\left.800 \mathrm{~cm}^{-1}\right)$ and $\mathrm{Si}-\mathrm{O}-\mathrm{Si}\left(1100 \mathrm{~cm}^{-1}\right)$ but no peaks attributed to $\mathrm{O}_{3} \mathrm{Si}-\mathrm{H}$ vibrations, ${ }^{3,33}$ see Figure $2 \mathrm{~A}$ (main figure). Therefore, a high degree of surface oxidation is observed for HOSi-NPB. A mean diameter of $3 \pm 1 \mathrm{~nm}$ (Figure 4) was determined by TEM.

Surface Functionalized Blue-Emitting Particles. The FTIR spectrum for the blue-emitting nanoparticles functionalized with methanol (MeSi-NPB) supported on a silicon wafer (Figure 2B, top spectrum) shows a set of peaks around 2920 and $2850 \mathrm{~cm}^{-1}$ due to $\mathrm{C}-\mathrm{H}$ stretching of the $\mathrm{CH}_{3}$ group, and a very small absorption at $1470 \mathrm{~cm}^{-1}$ attributed to $\mathrm{Si}-\mathrm{C}$ scissoring. ${ }^{1,34}$ The broad absorption with a maximum around $1050 \mathrm{~cm}^{-1}$ due to polar $\mathrm{Si}-\mathrm{O}-\mathrm{Si}$ bonds ${ }^{29}$ indicates some surface oxidation. No

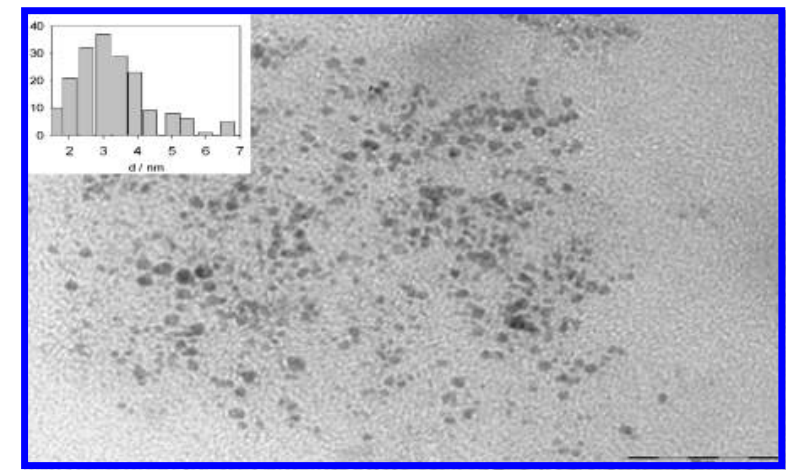

Figure 4. Representative TEM micrograph of HOSi-NPB nanoparticles. Scale bar: $50 \mathrm{~nm}$. Inset: Particle size histogram.

evidence for an $\mathrm{OH}$ stretching mode in the $3500-3000 \mathrm{~cm}^{-1}$ region was observed, indicating the absence of physisorbed methanol and of surface $\mathrm{Si}-\mathrm{OH}$ groups. Therefore, the covalent attachment of $\mathrm{CH}_{3}$ groups to the surface of the H-terminated $\mathrm{Si}-\mathrm{NPs}$ due to condensation reaction 1 is supported.

$$
\text { 到- } \mathrm{H}+\mathrm{CH}_{3} \mathrm{OH} \rightarrow \quad \text { 泾- } \mathrm{CH}_{3}+\mathrm{H}_{2} \mathrm{O}
$$

The ATR-IR spectrum of the methyl 2-methylprop-2-enoate functionalized nanoparticles (MMASi-NPB) using as a reference the $\mathrm{Si}$ wafer (bottom spectrum in Figure $2 \mathrm{~B}$ ) shows peaks at 2920 and $2850 \mathrm{~cm}^{-1}$ due to $\mathrm{C}-\mathrm{H}$ stretching of the $\mathrm{CH}_{3}$ group, at $1724 \mathrm{~cm}^{-1}$ due to the $\mathrm{C}=\mathrm{O}$ stretching in esters, and at 1450 and $1370 \mathrm{~cm}^{-1}$ due to the contribution of $\mathrm{CH}_{3}$ deformation modes in alkanes and esters, respectively. The absorption at $1260 \mathrm{~cm}^{-1}$ is assigned to $\mathrm{Si}-\mathrm{CH}_{2}-\mathrm{CH}$ deformation, and those at 1600 and $960 \mathrm{~cm}^{-1}$ may be attributed to $\mathrm{Si}-\mathrm{C}=\mathrm{C}$ vibration modes. ${ }^{35}$ The latter bands together with the absence of signals at $2100-2200 \mathrm{~cm}^{-1}$ due to $\mathrm{Si}-\mathrm{H}$ suggest a successful covalent attachment of methyl ester linkers to the surface, as shown in reaction 2 for two proposed structures. The presence of peaks at 1115 and $1055 \mathrm{~cm}^{-1}$ characteristic of $\mathrm{Si}-\mathrm{O}-\mathrm{Si}$ in small particles (and probably that at $\left.830 \mathrm{~cm}^{-1}\right)^{27,36}$ is indicative of a surface oxidized silicon particle, though the absence of bands around $3500 \mathrm{~cm}^{-1}$ clearly indicates the lack of $\mathrm{Si}-\mathrm{OH}$ bonds. Successful methyl 2-methylprop-2-enoate attachment is also supported by the determination of contact angles $>120^{\circ}$ for water drops on the Si-NP surface, suggesting a hydrophobic NP surface, compared to contact angles $<90^{\circ}$ observed for the hydrophilic silicon wafer surface. ${ }^{34}$

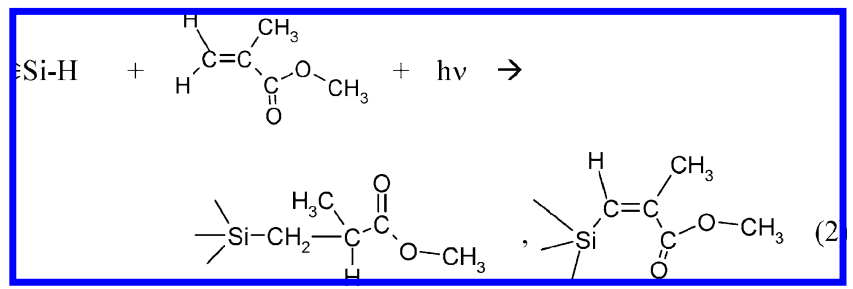

The XPS spectrum obtained for MMASi-NPB shows signals due to $\mathrm{O} 1 \mathrm{~s}, \mathrm{C} 1 \mathrm{~s}, \mathrm{Si} 2 \mathrm{~s}$, and $\mathrm{Si} 2 \mathrm{p}$ surface elements. The Si2p region (see Figure 5A) mainly displays the contribution of a peak at $102.6 \mathrm{eV}$ (1.6 fwhm) which shows a local environment of silicon between those of $\mathrm{Si}-\mathrm{C}$ and $\mathrm{Si}-\mathrm{O}$ bonds, reported at 101 and $103.5 \mathrm{eV}$, respectively. A peak at $102.5 \mathrm{eV}$ was assigned to $\mathrm{Si}(\mathrm{C}, \mathrm{O})$ environments in polymethylsilsesquioxanes ${ }^{37}$ and in oxycarbide glasses, ${ }^{38}$ and to $\mathrm{Si}-\mathrm{SiO}_{3}$ environments 


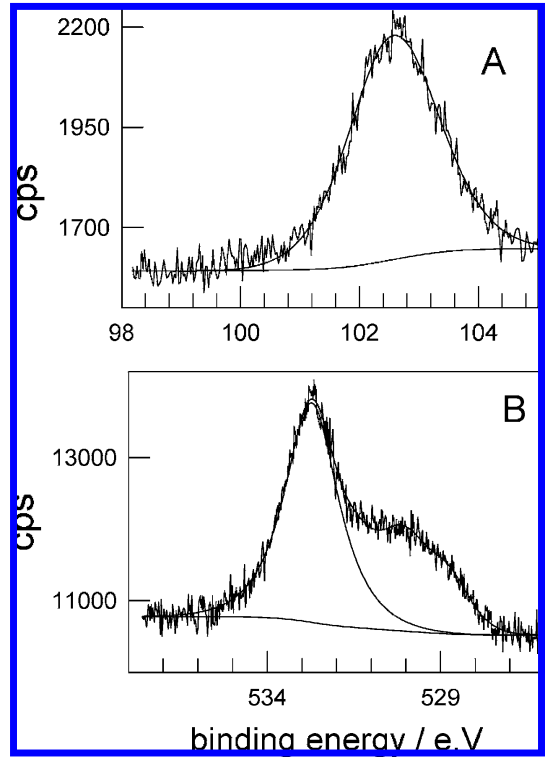

Figure 5. (A) Si2p and (B) O1s XPS peaks observed for MMASiNPB.

in $\mathrm{SiO}$ films. ${ }^{39}$ The O1s region shown in Figure 5B displays the contribution of three different environments for the oxygen atoms. The most important contribution (67\%) with a binding energy of $532.7 \mathrm{eV}$ could be attributed to $\mathrm{Si}-\mathrm{O}$ bonds. ${ }^{39}$ The ratio of areas of the latter peak to that of Si2p yields approximately a $1: 2 \mathrm{Si}: \mathrm{O}$ ratio. Assuming that the remaining oxygen atoms belong to the ester group of the surface attached organic molecule, the ratio between the areas of these oxygen atoms and that of silicon indicates the attachment of one organic molecule for every three silicon atoms.

Therefore, both the FTIR and XPS data agree in indicating that the particle surface is highly oxidized with a layer of $\mathrm{SiO}_{2}$ and that surface functionalization with MMA leads to the formation of surface $-\mathrm{O}-\mathrm{Si}-\mathrm{C}-$ networks. On average, the $\mathrm{Si}: \mathrm{O}: \mathrm{C}$ ratio at the surface of the derivatized particles seems to be $\mathrm{Si}_{3} \mathrm{O}_{6}\left(\mathrm{C}_{5} \mathrm{O}_{2} \mathrm{H}_{y}\right)_{1}$, with $y=7-8$. A mean diameter of the order of $2.5 \pm 0.5 \mathrm{~nm}$ was determined by TEM for MMASi-NPB.

Photophysical Properties. Figure 6 shows the absorption, emission, and excitation spectra of toluene suspensions of HOSiNPB, MeSi-NPB, and MMASi-NPB. The small differences observed in the relative intensity of the bands depends more on the synthesis batch than on the type of surface groups (see the Supporting Information for the spectra obtained for suspensions of different batches of particles). The excitation spectra of the different particles show a common maximum. However, some fluctuations on the UV range of the excitation spectra may be observed with different Si-NP batches and different surface groups; see Figure 6B. Solvent evaporation and further dispersion of the MMASi-NPB particles in methanol, ethanol, chloroform, carbon tetrachloride, or toluene does not change, within the experimental error, the particle PL properties. PL was stable in ambient air for up to 4 months. Dispersion in water considerably diminishes the particle emission and causes a loss of the peak resolution of the emission spectrum.

Emission and excitation in the same spectral region was also reported for $5 \mathrm{~nm}$ partially surface oxidized organically capped Si-NPs ${ }^{11}$ and for $3-4 \mathrm{~nm}$ octylcapped Si-NPs. ${ }^{13}$ However, despite the fact that the emission is in the same wavelength region, the spectrum and its dependence on the excitation wavelength differ from those reported here.

Both the emission and excitation spectra show peaks with a constant spacing of approximately $0.2 \mathrm{eV}$, as shown by the

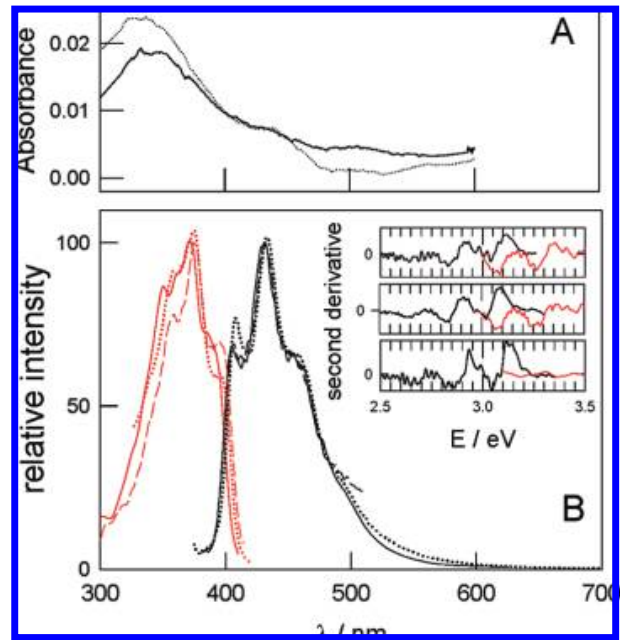

Figure 6. (A) Absorption spectra (uncorrected for scattering) of argonsaturated toluene suspensions of MMASi-NPB (full line) and HOSiNPB (dashed line). (B) Excitation (red) and emission (black) spectra of toluene suspensions of HOSi-NPB (dashed lines), MeSi-NPB (full line), and MMASi-NPB (dotted line). Inset: Second derivative of the emission and excitation spectra of the main figure. From top to bottom: MeSi-NPB, MMASi-NPB, and HOSi-NPB.

separation between minima in the second derivative spectra displayed in Figure 6B, inset. A peak structure in the photoluminescence of electrochemically synthesized Si-NPs was previously reported ${ }^{40,41}$ and attributed either to the contribution of $\mathrm{Si}$ nanocrystals with a specific number of atoms and surface configuration ${ }^{40}$ or to the presence of oxygen on the particle surface ${ }^{41}$ The fine peak structure was not affected by the length of the alkyl chain of the surface attached hydrocarbons nor by the variation of the excitation wavelength ${ }^{40}$ in coincidence with our observations, but showed excitation-wavelength-dependent relative emission intensity. Peak resolution in the absorption and emission spectrum of nanometer size Si-NPs synthesized by chemical reaction of Si precursors in solution was attributed to the participation of vibrations of silicon-bonded surface groups due to the surface localization of the lowest unoccupied molecular orbital (LUMO). ${ }^{1}$ As a consequence, electronic excitation of the NPs yields a shift in the electron density toward the surface of the NPs, with a concomitant change in geometry. As discussed above, HOSi-NPB, MeSi-NPB, and MMASi-NPB are covered by surface siloxane networks and, except for HOSiNPB, do not show vibrations of $0.2 \mathrm{eV}$ in their IR spectrum (IR peaks at $1630 \mathrm{~cm}^{-1}$ ). We therefore suggest that $\mathrm{Si}-\mathrm{SiO}_{2}$ networks may either induce specific surface configurations or else undergo multiple vibrations of negligible IR absorption leading to the observed peak resolution. The red luminescence observed for the bigger particles does not show vibrational features, as expected for larger systems where the wave functions approach those of simple quantum confinement independent of the oxidation of the surface. ${ }^{2}$

From the absorption and emission spectra, a fwhm of $\sim 70$ $\mathrm{nm}$ and a Stokes shift due to the energy difference of $\sim 0.47$ $\mathrm{eV}$ between the absorption and emission maximum peaks are determined. Theoretical and experimental studies on $\mathrm{CdS}$ and CdSe NPs indicate that particles with broad emission and large Stokes shift are considered to exhibit emission originated from the recombination of holes and trapped electrons due to surface irregularities. ${ }^{42}$ Both the latter observations and the peak resolution of the excitation and emission spectra indicate that the luminescence of the synthesized Si-NPs is dominated by surface interactions which are present in underivatized and derivatized NPs. 
TABLE 1: Room Temperature Quantum Yield, $\boldsymbol{\Phi}$, of Air-Saturated Toluene Suspensions of MMASi-NPB and HOSi-NPB as a Function of the Excitation Wavelength, $\lambda_{\mathrm{exc}}{ }^{a}$

\begin{tabular}{cccccc}
\hline & \multicolumn{2}{c}{ MMASi-NPB } & & \multicolumn{2}{c}{ HOSi-NPB } \\
\cline { 2 - 3 } \cline { 5 - 6 }$\lambda_{\text {exc }} / \mathrm{nm}$ & $\Phi$ & $\Phi_{\text {corr }}$ & & $\Phi$ & $\Phi_{\text {corr }}$ \\
\hline 340 & $0.28 \pm 0.04$ & $0.40 \pm 0.04$ & & $0.19 \pm 0.03$ & $0.26 \pm 0.03$ \\
350 & $0.34 \pm 0.05$ & $0.43 \pm 0.05$ & & $0.22 \pm 0.03$ & $0.27 \pm 0.03$ \\
360 & $0.41 \pm 0.06$ & $0.45 \pm 0.06$ & & $0.25 \pm 0.04$ & $0.27 \pm 0.04$ \\
370 & $0.48 \pm 0.07$ & $0.48 \pm 0.07$ & & $0.28 \pm 0.04$ & $0.28 \pm 0.04$
\end{tabular}

${ }^{a}$ Corrected quantum yields, $\Phi_{\text {corr }}$, assume a $\left(\lambda_{\text {exc }} / 370\right)^{-4}$ correction for the dispersion of light (see text).

To determine the quantum yields of luminescence, $\Phi, 9,10$ diphenylantracene in cyclohexane $\left(\Phi_{R}=0.90 \pm 0.04\right)$ was used as a reference emitter. ${ }^{20} \mathrm{~A}$ comparison of the ratios between the area of the emission spectrum and the absorbance at a given excitation wavelength yields the quantum efficiency of MMASiNPs. ${ }^{43}$ Table 1 shows the values of $\Phi$ obtained at different excitation wavelengths for Si-NPB and MMASi-NPB in airsaturated suspensions. The measured quantum yields of the latter particles decrease with decreasing wavelengths, as also observed for $1 \mathrm{~nm}$ alkyl-capped Si-NPs. ${ }^{1}$ Scattering of light by NPs causes the measured attenuance to be higher than the actual absorbance, as the scattered part of the incoming light is recorded as absorption but does not necessarily excite the sample and therefore it does not result in any recorded emission. Scattering depends on the wavelength of the incoming light as $\lambda^{-4}$ and is proportional to the square of the particle volume ${ }^{42}$ Assuming a $(\lambda / 370)^{-4}$ correction for the dispersion of light, the corrected quantum yields, $\Phi_{\text {corr }}$, also listed in Table 1 , show, within the experimental error, no dependence on the excitation wavelength. A wavelength-independent quantum yield is expected for an excitonic transition, as is the case for fluorescent molecules with emission from only one excited state. The latter considerations are in agreement with the observation of mainly one luminescence decay time (vide infra) and an emission spectrum independent of the excitation wavelength. The measured values are on the order reported for other Si-NPs, despite their different crystallinity. ${ }^{12,13}$

Higher quantum yields are observed for MMASi-NPB than for HOSi-NPB, which therefore indicates that the particle photoluminescence is not quenched by surface derivatization with MMA.

Luminescence time-resolved experiments indicate a rapid relaxation of electronically excited Si-NPs to the ground state. The modulation and phase curves obtained from the frequencydomain experiments of air-saturated suspensions of the particles may be well fitted if two decay lifetime components are considered (see Figure 7A). The fitting parameters obtained for HOSi-NPB curves suggest a short lifetime component of 1.1 $\pm 0.1 \mathrm{~ns}$ contributing to $78-88 \%$ of the total luminescence, and a longer component of $5.8 \pm 0.3 \mathrm{~ns}$ responsible for the remaining $12-22 \%$ of the emitted light. MMASi-NPB parameters indicate almost the same lifetimes, $1.1 \pm 0.1 \mathrm{~ns}$ with a $95-97 \%$ contribution to the emitted light, and a very small contribution of a longer component of $4.6 \pm 0.4 \mathrm{~ns}$. Since both underivatized and MMA derivatized particles were obtained from the same synthetic batch, the differences in size distribution and surface configuration are expected to be negligible. However, HOSi-NPBs are observed to agglomerate with aging. The observation of almost one lifetime component for MMASi-NPs seems to be in line with the fact that these particles are less prone to agglomeration, as also indicated by the long stability of their suspensions (vide supra). Particle agglomeration may

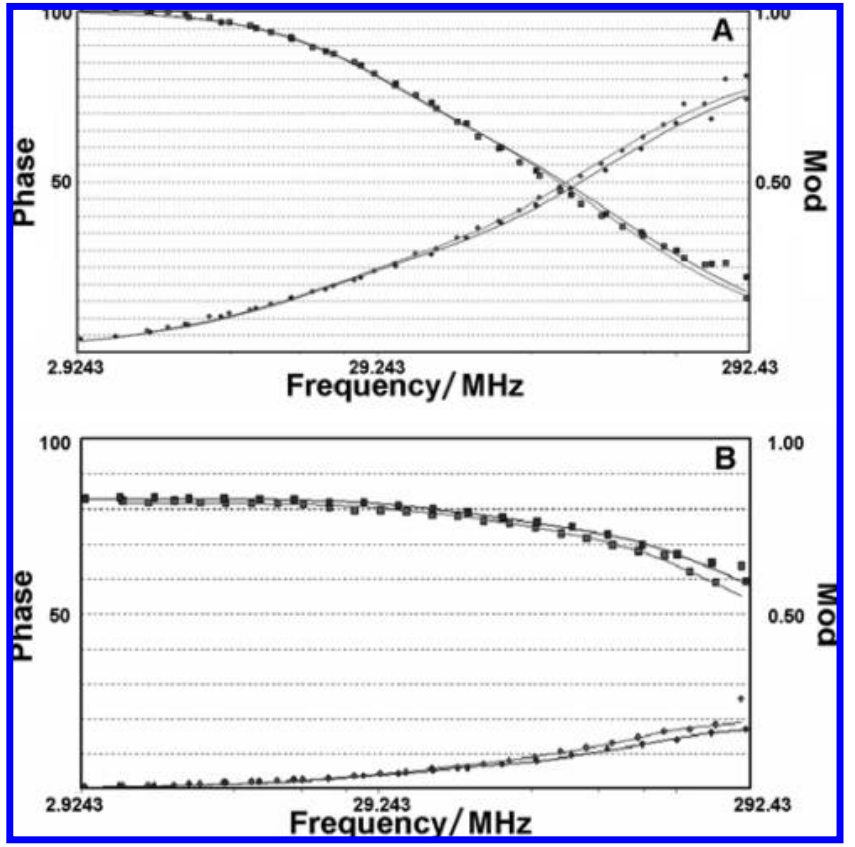

Figure 7. Frequency-domain experiments performed for air-saturated toluene suspensions of HOSi-NPs: (A) luminescence decay; (B) luminescence decay anisotropy measurements. Each figure shows the results of two experiments. Circles and squares stand for phase and modulation curves, respectively.

TABLE 2: Anisotropy at Time Zero $\left(\mathbf{r}_{0}\right)$ and Rotational Correlation Times $(\boldsymbol{\theta})$ Obtained from Frequency-Domain Experiments Performed for Air-Saturated Toluene Suspensions of HOSi-NPB and MMASi-NPB

\begin{tabular}{lcc}
\hline & $\mathbf{r}_{0}$ & $\theta / \mathrm{ns}$ \\
\hline HOSi-NPB & $0.25 \pm 0.02$ & $0.62 \pm 0.01$ \\
MMASi-NPB & $0.26 \pm 0.01$ & $0.25 \pm 0.01$
\end{tabular}

also be a cause for the lower quantum yields observed for HOSiNPB suspensions.

The shorter component, responsible for most parts of the emission, supports a direct band gap transition. In fact, the general observations on the photophysical properties of the particles show some similarity with early publications on $\mathrm{PS}^{44}$ which report transformation of PS $\mu$ s red luminescence into fast $1-10$ ns blue luminescence (centered at $480 \mathrm{~nm}$ ) after thermal and chemical surface oxidation.

Anisotropy frequency-domain experiments, through the determination of the rotational correlation times $\left(\theta_{i}\right)$ and the anisotropy at time zero $\left(\mathbf{r}_{0}\right)$, give information on the shape of the particles and on the relative orientation of the excited and fundamental state dipole moments of the particles. These experiments show that the anisotropy of HOSi-NPB and MMASi-NPB follows a single exponential anisotropy decay, $r(t)=\mathbf{r}_{0} \exp (t / \theta)$; see Figure 7B for a typical experiment. This can only be the case if the emission originates mainly from one population of particles with identical three perpendicular rotational axes. This indicates that Si-NPs are ball-shaped, as also observed by TEM. Table 2 summarizes the results obtained. Both particles show, within the experimental error, the same $\mathbf{r}_{0}$ which therefore indicates that the dipole orientation of the excited states leading to the observed luminescence does not depend on surface coverage. The value $\mathbf{r}_{0}=0.25$ clearly indicates a different orientation of the fundamental and excited state dipole moments, also in agreement with the large Stokes shift between the excitation and emission spectra. A similar value was obtained for CdS/dendrimer NPs. ${ }^{43}$ 


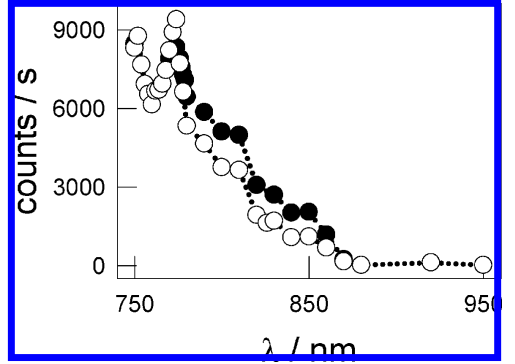

Figure 8. Biphotonic excitation spectrum of air-saturated toluene suspensions of HOSi-NPB $(\bullet)$ and MMASi-NPB $(O)$.

The dependence of $\theta$ on the volume $(V)$ for spherical particles, given by the simple hydrodynamic theory of the rotational diffusion is $\theta=\eta V / k_{\mathrm{B}} T$, where $k_{\mathrm{B}}$ is the Boltzmann constant and $\eta=554.2 \mu$ Pas is the shear viscosity of toluene at $298 \mathrm{~K}$. From this relation, a mean ratio of $1.1 \mathrm{~nm}$ and of $0.8 \mathrm{~nm}$ is predicted for HOSi-NPB and MMASi-NPB, respectively. Since mainly HOSi-NPB suspensions show agglomeration, and these suspensions show a large lifetime component contributing around $12-22 \%$ to the total emitted light, the larger value observed for the mean radius of these particles is probably due to the contribution of agglomerated particles.

The biphotonic excitation spectrum of HOSi-NPB and MMASi-NPB was also measured to obtain further information on their potential use as luminescence markers in biological systems, as the use of near-infrared radiation in a two-photon process is less damaging to the sample. This spectrum provides better discrimination against scattered incident radiation, as well as better definition of the probed volume. ${ }^{45}$ A blue emission is observed when toluene suspensions of MMASi-NPB and of HOSi-NPB are excited by the pulsed laser in the $750-880 \mathrm{~nm}$ range in a two-photon process. The corresponding excitation spectra are shown in Figure 8.

In multiphoton excitation processes, higher intensities are needed to induce excitation; thus, the effective excitation volume is restricted to the central portion of the focal spot where the intensity is the highest, allowing fluctuating signals for nanomolar solutions of fluorophores to be detected. Under these conditions, measurements of the photon counts with time, $P(t)$, yield luminescence fluctuations, $\delta P(t)$, around the mean value that are autocorrelated with time. The luminescence correlation function $G(\tau)$ contains the information of amplitude of fluctuations as well as of their persistence, which yield information on size, brightness, and number density. ${ }^{45}$ For a single species, the autocorrelation function at $\tau=0, G(0)$, is proportional to the inverse of the average number of the emitting species in the focal volume. Table 3 depicts the results of the analysis of the autocorrelation functions obtained for the different experimental conditions.

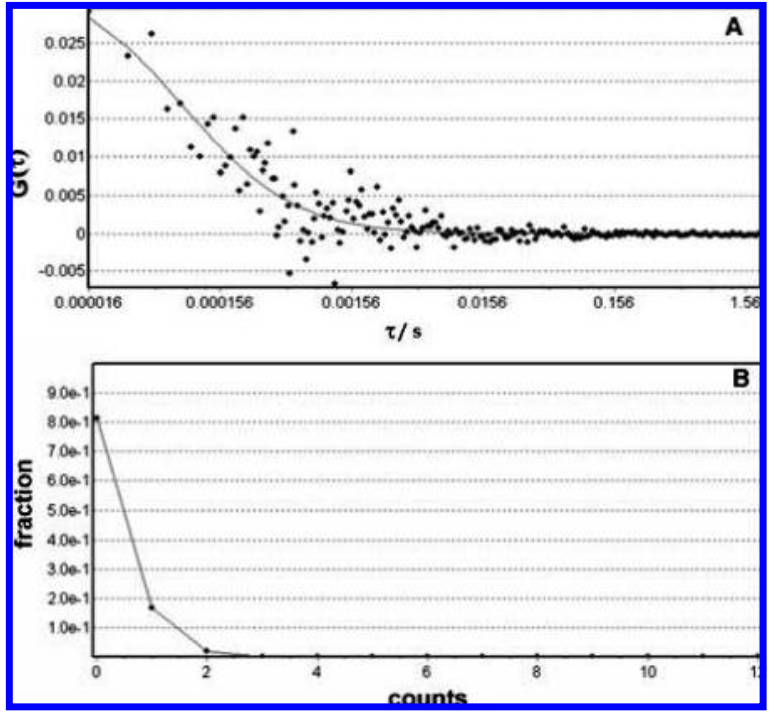

Figure 9. (A) Luminescence autocorrelation observed for air-saturated toluene suspensions of MMASi-NPB obtained exciting with light of $826 \mathrm{~nm}$. (B) Photon correlation histogram $(\mathrm{PCH})$ obtained from the experiments in part $\mathrm{A}$.

The luminescence correlation observed for HOSi-NPB upon $774 \mathrm{~nm}$ irradiation may be well fitted to one diffusing species with an average diffusion coefficient of $D=(150 \pm 20) \times$ $10^{-12} \mathrm{~m}^{2} / \mathrm{s}$. Experiments with 1:10 diluted suspensions show similar $D$ values but lower $1 / G(0)$ values (see Table 3 ).

The autocorrelation functions for MMASi-NPB were obtained by exciting at different wavelengths. Except for experiments at $774 \mathrm{~nm}$, all $G(\tau)$ curves could be well fitted to one diffusing species (see Figure 9A for a typical curve) with an average diffusion coefficient of $(240 \pm 40) \times 10^{-12} \mathrm{~m}^{2} / \mathrm{s}$ in toluene. At $774 \mathrm{~nm}$, correlation curves could only be fitted if two different diffusing species are assumed. Because the different behavior was only observed for $774 \mathrm{~nm}$, these data were not used for further calculations.

According to our previous results (vide supra), the particles may be assumed spherical. Therefore, the Stokes-Einstein equation was used for the calculation of the hydrodynamic radius of the particle taking $\eta=554.2 \mu$ Pas for the viscosity of toluene at $298 \mathrm{~K}$; see Table 3 . An average radius of $1.5 \pm 0.2 \mathrm{~nm}$ is obtained for MMASi-NPB, in agreement with the value measured by TEM (vide supra). A mean hydrodynamic radius of $2.6 \pm 0.3 \mathrm{~nm}$ is obtained for HOSi-NPB, higher than that of the derivatized particles. The trend observed for the average radius of HOSi-NPB and MMASi-NPB particles is in agreement with those obtained from the luminescence anisotropy experiments, further supporting the contribution of agglomerated

TABLE 3: PCH and Fluorescence Correlation Obtained for HOSi-NPB and MMASi-NPB under Different Experimental Conditions $^{a}$

\begin{tabular}{|c|c|c|c|c|c|c|}
\hline & $\begin{array}{l}\text { excitation wavelength } \\
\qquad(A) / \mathrm{nm}\end{array}$ & $\begin{array}{l}\text { molecular brightness/counts } \\
\qquad \mathrm{s}^{-1} \text { molecule }^{-1}\end{array}$ & $\langle N\rangle$ & $1 / G(0)$ & $D / \mu \mathrm{m}^{2} \mathrm{~s}^{-1}$ & $r / \mathrm{nm}$ \\
\hline HOSi-NP & $774.0(1)$ & 0.176 & 1.0 & 500 & 172 & 2.3 \\
\hline 1/10 diluted & $774.0(1)$ & 0.176 & 0.09 & 22 & 136 & 2.9 \\
\hline \multirow[t]{5}{*}{ MMASi-NP } & $750.0(0.54)$ & 0.094 & 1.6 & $150 \pm 50$ & $120 \pm 80$ & $3.3 \pm 1$ \\
\hline & $800.0(0.54)$ & 0.093 & 1.2 & 93.5 & 392.5 & 1.0 \\
\hline & $826.0(0.54)$ & 0.028 & 1.2 & $15 \pm 0.5$ & $245 \pm 2$ & 1.6 \\
\hline & $840.0(0.54)$ & 0.023 & 1.0 & 23.5 & 206 & 1.9 \\
\hline & $860.0(0.54)$ & 0.024 & 1.0 & 11 & 246 & 1.6 \\
\hline rhodamine 110 & $800(0.54)$ & 0.131 & 0.75 & & & \\
\hline
\end{tabular}

${ }^{a} G(0)$ is the fluorescence correlation at $\tau=0$. "A" stands for the absorbance of the filter on the excitation pathway. 
particles in the suspensions of HOSi-NPB used for these experiments.

The analysis of amplitudes of the luminescence fluctuations $\delta P(t)$ is achieved by the photon counting histogram ( $\mathrm{PCH})$ method (frequency of observation vs photon counts), measured within a suitable time window of $\sim 8 \mathrm{~ms}$. The deviation of the histogram from the ideal Poisson distribution contains information on the average number of particles in the excitation volume $(\langle N\rangle)$ and on the molecular brightness $(\eta)$. Figure 9B shows the histogram obtained for a typical experiment, and Table 3 depicts the number density and brightness values obtained in experiments performed under different experimental conditions.

$\langle N\rangle$ defined as the product of $C \times V_{0} \times N_{\mathrm{A}}$ (where $C$ is the molar concentration of particles, $V_{0}$ the excitation volume, and $N_{\mathrm{A}}$ Avogadro's constant) is expected to be independent of the excitation wavelength. The variations observed in experiments with different excitation wavelengths but otherwise identical conditions may be due to a variation in $V_{0}{ }^{46}$

In analogy to the monophotonic brightness (the product of the absorption coefficient and the fluorescence quantum yield), the "brightness" of a fluorophore under two-photon excitation is a quantitative measure of the product $\sigma_{2} \times \Phi$, where $\sigma_{2}$ is the two-photon absorption cross section. Since the excited state from which emission takes place is identical for both one- and two-photon excitation, the fluorescence quantum yield may be assumed the same under one- and two-photon excitation. ${ }^{47}$ Therefore, brightness measurements yield information on the two-photon absorption cross sections of the NPs.

The measured brightness values are dependent on the intrinsic properties of the fluorophore and design of optics and are not affected by concentration. In fact, under identical setup excitation and emission conditions, the brightness obtained for suspensions of different HOSi-NPB concentrations are coincident, and the values of $\langle N\rangle$ are correlated with the suspension concentration, as shown in Table 3. To obtain absolute brightness values, rhodamine 110 was used as a standard and its PCH measured at $800 \mathrm{~nm}$ under identical experimental conditions to those used for the NPs. Assuming an absolute brightness of rhodamine 110 at $800 \mathrm{~nm}$ of $\sigma_{2} \Phi \cong 40 \mathrm{GM}$ (Göppert-Mayer units, $1 \mathrm{GM}=1 \times 10^{-50} \mathrm{~cm}^{4}$ s/photon), ${ }^{48}$ the measured brightness of 0.131 counts s $^{-1}$ molecule ${ }^{-1}$ is equivalent to 40 GM. Therefore, the brightness of 0.093 counts s$^{-1}$ molecule $^{-1}$ measured at $800 \mathrm{~nm}$ for MMASi-NPB corresponds to an absolute brightness of $28 \mathrm{GM}$ from which $\sigma_{2}{ }^{800 \mathrm{~nm}} \cong 64 \mathrm{GM}$ is obtained taking $\Phi \cong 0.44$ (vide supra), the quantum yield in air-saturated suspensions. The obtained $\sigma_{2}$ value is of the order of those reported for organic fluorophores. ${ }^{47,49}$

\section{Conclusions}

The results herein obtained from steady-state and timeresolved measurements (including the anisotropy studies) indicate that room temperature PL of Si-NPs of $3 \pm 1 \mathrm{~nm}$ diameter and surface composition $\mathrm{Si}_{3} \mathrm{O}_{6} \mathrm{R}$, synthesized by the electrochemical etching of silicon wafers, is not influenced by the nature of the surface groups for $\mathrm{R}: \mathrm{C}_{5} \mathrm{O}_{2} \mathrm{H}_{2}, \mathrm{CH}_{3}$, or $\mathrm{OH}$. However, functionalization with the organic groups lends the particles higher stability in suspension. Moreover, time-resolved anisotropy measurements, FCS, and TEM images reveal that the particles are ball-shaped and confirm the monodispersity of the species under study. The short lifetime of $1 \mathrm{~ns}$ observed for the decay of the luminescence is consistent with what would be expected from molecular species and therefore supports a direct band gap transition which could result from the rapid trapping of the excitation at the nanocrystal surface. The participation of surface irregularities in the emission originated from the recombination of holes and trapped electrons is further supported by the value of $\mathbf{r}_{0}$ and the large Stokes shift. The measured brightness and quantum yields are on the order of those observed for common organic fluorophores. The evidence obtained suggests that the particle size clearly determines the emission and excitation spectral region, as expected from quantum confinement, but the presence and extent of $\mathrm{Si}-\mathrm{SiO}_{3}$ species on the silicon networks seem crucial in determining the characteristics of the emission and probably that of the surface configuration, lending the particles a PL behavior similar to those of organic fluorophores.

The PL bands and Stokes shifts reported for $\mathrm{Si}_{29} \mathrm{H}_{24}$ nanoclusters were theoretically explained in terms of intrinsic molecular-like excited states of the ideal bulklike silicon configuration, and some of the bands were suggested to originate from similar particles with different surface reconstructions. ${ }^{9}$ Despite the fact that theoretical studies agree in predicting that the replacement of hydrogen atoms with oxygen atoms induces remarkable changes in the spectroscopic properties of the silicon dots, much work is still needed to understand the effect of surface oxidation on surface configuration and on the optical properties of small particles.

Acknowledgment. The frequency-domain fluorescence and anisotropy measurements and fluorescence correlation spectroscopy experiments reported in this paper were performed at the Laboratory for Fluorescence Dynamics (LFD) at the University of California, Irvine (UCI). The LFD is supported jointly by the National Center for Research Resources of the National Institutes of Health (PHS 5 P41-RR003155) and UCI. The authors thank Professor Silvia Braslavsky for her useful comments and discussions related to this work. Drs. Guillermo Benitez and Aldo Rubbert (INIFTA, University of La Plata, Argentina) are thanked for the XPS spectrum and Antonio Arques Sanz (Universidad Politécnica de Valencia, Spain) for obtaining the TEM images. This work has been supported by projects PIP 6301 and 1542/06 from Consejo Nacional de Investigaciones Científicas y Tecnológicas (CONICET), Argentina. M.C.G., M.K., and D.B.S. are research members of CONICET. F.R.N., D.O.M., and J.I.A. are research members of Comisión de investigaciones Científicas de la Provincia de Buenos Aires (CICPBA). M.J.L.P. thanks CONICET for a studentship.

Supporting Information Available: Figure showing the emission spectrum obtained exciting at $360 \mathrm{~nm}$ different HOSiNP and MMASi-NP batches. This material is available free of charge via the Internet at http://pubs.acs.org.

\section{References and Notes}

(1) Rosso-Vasic, M.; Spruijt, E.; Van Lagen, B.; De Cola, L.; Zuilhof, H. Alkyl-functionalized oxide-free silicon nanoparticles: synthesis and optical properties. Small 2008, 4, 1835-1841.

(2) Wolkin, M. V.; Jorne, J.; Fauchet, P. M.; Allan, G.; Delerue, C. Electronic states and luminescence in porous silicon quantum dots: the role of oxygen. Phys. Rev. Lett. 1999, 82, 197-200.

(3) Belomoin, G.; Therrien, J.; Nayfeh, M. Oxide and hydrogen capped ultrasmall blue luminescent Si nanoparticles. Appl. Phys. Lett. 2000, 77, 779-781.

(4) Choi, J.; Wang, N. S.; Reipa, V. Photoassisted tuning of silicon nanocrystal photoluminescence. Langmuir 2007, 23, 3388-3394.

(5) Michalet, X.; Pinaud, F. F.; Bentolila, L. A.; Tsay, J. M.; Doose, S.; Li, J. J.; Sundaresan, G.; Wu, A. M.; Gambhir, S. S.; Weiss, S. Quantum dots for live cells, in vivo imaging, and diagnostics. Science 2005, 307, $538-544$. 412. 
(7) Bley, R. A.; Kauzlarich, S. M.; Davis, J. E.; Lee, H.W. H. Characterization of silicon nanoparticles prepared from porous silicon. Chem. Mater. 1996, 8, 1881-1888.

(8) Rogozhina, E. V.; Eckhoff, D. A.; Gratton, E.; Braun, P. V. Carboxyl functionalization of ultrasmall luminescent silicon nanoparticles through thermal hydrosilylation. J. Mater. Chem. 2006, 16, 1421-1430.

(9) Rao, S.; Sutin, J.; Clegg, R.; Gratton, E.; Nayfeh, M. H.; Habbal, S.; Tsolakidis, A.; Martin, R. M. Excited states of tetrahedral single-core Si29 nanoparticles. Phys. Rev. B 2004, 69, 205319.

(10) Liu, S. M. Luminescent silicon nanoparticles formed in solution. J. Nanosci. Nanotechnol. 2008, 8, 1110-1125.

(11) Hua, F.; Erogbogbo, F.; Swihart, M. T.; Ruckenstein, E. Organically capped silicon nanoparticles with blue photoluminescence prepared by hydrosilylation followed by oxidation. Langmuir 2006, 22, 4363-4370.

(12) Sankaran, R. M.; Holunga, D.; Flagan, R. C.; Giapis, K. P. Synthesis of blue luminescent $\mathrm{Si}$ nanoparticles using atmospheric-pressure microdischarges. Nano Lett. 2005, 5, 537-541.

(13) Zhang, X.; Neiner, D.; Wang, S.; Louie, A. Y.; Kauzlarich, S. M. A new solution route to hydrogen-terminated silicon nanoparticles: synthesis, functionalization and water stability. Nanotechnology 2007, 18, 095601.

(14) Pradhan, S.; Chen, S.; Zou, J.; Kauzlarich, S. M. Photoconductivity of Langmuir-Blodgett monolayers of silicon nanoparticles. J. Phys. Chem. C 2008, 112, 13292-13298.

(15) Kanemitsu, Y. Efficient light emission from crystalline and amorphous silicon nanostructures. J. Lumin. 2002, 100, 209-217.

(16) Mitas, L.; Therrien, J.; Twesten, R.; Belomoin, G.; Nayfeh, M. H. Effect of surface reconstruction on the structural prototypes of ultrasmall ultrabright Si29 nanoparticles. Appl. Phys. Lett. 2001, 78, 1918-1920.

(17) Zhou, Z.; Brus, L.; Friesner, R. Electronic structure and luminescence of 1.1- and 1.4-nm silicon nanocrystals: Oxide shell versus hydrogen passivation. Nano Lett. 2003, 3, 163-167.

(18) Yamani, Z.; Thompson, W. H.; AbuHassan, L.; Nayfeh, M. H. Ideal anodization of silicon. Appl. Phys. Lett. 1997, 70, 3404-3406.

(19) Valenta, J.; Janda, P.; Dohnalova, K.; Nizňănsky, D.; Vácha, F.; Linnros, J. Colloidal suspensions of silicon nanocrystals: from single nanocrystals to photonic structures. Opt. Mater. 2005, 27, 1046-1049.

(20) Li, X.; He, Y.; Swihart, M. T. Surface functionalization of silicon nanoparticles produced by laser-driven pyrolysis of silane followed by HF$\mathrm{HNO}_{3}$ etching. Langmuir 2004, 20, 4720-4727.

(21) Hua, F.; Erogbogbo, F.; Swihart, M. T. Ruckenstein. Efficient surface grafting of luminescent silicon quantum dots by photoinitiated hydrosilylation. Langmuir 2005, 21, 6054-6062.

(22) Hamal, S.; Hirayama, F. Actinometric Determination of Absolute Fluorescence Quantum Yields. J. Phys. Chem. 1983, 87, 83-89.

(23) Scurlock, R. D.; Ogilby, P. R. Singlet molecular oxygen $\left(1 \Delta_{\mathrm{g}} \mathrm{O}_{2}\right)$ formation upon irradiation of an oxygen $\left(3 \sum_{\mathrm{g}}{ }^{-} \mathrm{O}_{2}\right)$-organic molecule charge -transfer absorption band. J. Phys. Chem. 1989, 93, 5493-5500.

(24) Mueller, J. D.; Gratton, E. High -Pressure Fluorescence Correlation Spectroscopy. Biophys. J. 2003, 85, 2711-2719.

(25) Valeur, B. Molecular Fluorescence. Principles and applications. Angew. Chem., Int. Ed. Engl. 2002, 41, 3063-3064.

(26) San Roman, E.; Gonzalez, M. C. Photochemistry of aqueous solutions of triphenyltetrazolium chloride. J. Phys. Chem. 1989, 93, 35363540 .

(27) Kravetz, V. G.; Meier, C.; Konjhodzic, D.; Lorke, A. Infrared properties of silicon nanoparticles. J. Appl. Phvs. 2005, 97, 084306.

(28) Li, Z. F.; Swihart, M. T.; Ruckenstein, E. Luminescent silicon nanoparticles capped by conductive polyaniline through the self-assembly method. Langmuir 2004, 20, 1963-1971.

(29) Bateman, J. E.; Horrocks, B. R.; Houlton, A. Reactions of water and methanol at hydrogen-terminated silicon surfaces studied by transmission FTIR. J. Chem. Soc. 1997, 93, 2427-2431.
(30) Sweryda-Krawiec, B.; Cassagneau, T.; Fendler, J. H. Modification of silicon nanocrystallites by alcohols. J. Phvs. Chem. B 1999, 103, 95249529.

(31) Kim, N. Y.; Laibinis, P. E. Improved polypyrrole/ silicon junctions by surfacial modification of hydrogen-terminated silicon using organolithium reagents. J. Am. Chem. Soc. 1999, 121, 7162-7163.

(32) Ruiz, A. E.; Caregnato, P.; Arce, V. B.; Schiavoni, M. M.; Mora, V. C.; Gonzalez, M. C.; Allegretti, P. E.; Mártire, D. O. Synthesis and characterization of butoxylated silica nanoparticles. reaction with benzophenone triplet states. J. Phys. Chem. C 2007, 111, 7623-7628.

(33) Ma, L. B.; Ji, A. L.; Liu, C.; Wang, Y. Q.; Cao, Z. X. Low temperature growth of amorphous $\mathrm{Si}$ nanoparticles in oxide matrix for efficient visible photoluminescence. J. Vac. Sci. Technol., B 2004, 22, 2654 2657.

(34) Sun, Q.-Y.; Louis, C. P. M.; de Smet; Van Lagen, B.; Giesbers, M.; Thne, P. C.; Van Engelenburg, J.; de Wolf, F. A.; Zuilhof, H.; Sudhlter, E.J. R. Covalently attached monolayers on crystalline hydrogen-terminated silicon: extremely mild attachment by visible light. J. Am. Chem. Soc. 2005, 127, 2514-2523.

(35) Larson, G. L., Arkles B.; Anderson R. Silicon compounds, register and review, Petrarch Systems, 1987.

(36) Chung, T. H. Effects of process parameters on the properties of silicon oxide films using plasma enhanced chemical vapor deposition with tetramethoxysilane. Curr. Appl. Phys. 2009, 9, 598-604.

(37) Jum, M. A.; Feng, Q. Y.; Shi, L. H.; Xu, J. Preliminary study on pyrolysis of polymethylsilsesquioxane by FT-IR and XPS. Chin. Chem. Lett. 2002, 13, 75-78.

(38) Sorarú, G. D.; Andrea, G. D.; Glisenti, A. XPS characterization of gel-derived silicon oxycarbide glasses. Mater. Lett. 1996, 27, 1-5.

(39) Nguyen, T. P.; Lefrant, S. XPS study of SiO thin films and SiOmetal interfaces. J. Phys.: Condens. Matter 1989, 5197-5204.

(40) Dobrovolskas, D.; Mickevičius, J.; Tamulaitis, G.; Reipa, V. Photoluminescence of Si nanocrystals under selective excitation. J.Phys. Chem. Solids 2009, 70, 439-443.

(41) Holmes, J. D.; Ziegler, K. J.; Doty, R. C.; Pell, L. E.; Johnston, K. P.; Korgel, B. A. Highly luminescent silicon nanocrystals with discrete optical transitions. J. Am. Chem. Soc. 2001, 123, 3743-3748.

(42) Landes, C. F.; Link, S.; Mohamed, M. B.; Nikoobakht, B.; ElSayed, M. A. Some properties of spherical and rod-shaped semiconductor and metal nanocrystals. Pure Appl. Chem. 2002, 74, 1675-1692.

(43) Lakowicz, J. R.; Gryczynski, I.; Gryczynski, Z.; Murphy, C. J. Luminescence spectral properties of CdS nanoparticles. J. Phys. Chem. B 1999, 103, 7613-7620.

(44) Tsybeskov, L.; Vandeshev, J. V.; Fauchet, P. M. Blue emission in porous silicon: oxygen-related photoluminescence. Phys. Rev. B 1994, 49, R7821.

(45) Akcakir, O.; Therrien, J.; Belomoin, G.; Barry, N.; Muller, J. D.; Gratton, E.; Nayfeh, M. Detection of luminescent single ultrasmall silicon nanoparticles using fluctuation correlation spectroscopy. Appl. Phys. Lett. 2000, 76, 1857-1859.

(46) Chirico, G.; Bettati, S.; Mozzarelli, A.; Chen, Y.; Mueller, J. D.; Gratton, E. Molecular heterogeneity of $O$-acetylserine sulfhydrylase by twophoton excited fluorescence fluctuation spectroscopy. Biophys. J. 2001, 80, 1973-1985.

(47) Lebret, V.; Raehm, L.; Durand, J.; Smaïhi, M.; Gérardin, C.; Nerambourg, N.; Werts, M. H. V.; Blanchard-Desce, M. Synthesis and characterization of fluorescently doped mesoporous nanoparticles for twophoton excitation. Chem. Mater. 2008, 20, 2174-2183.

(48) Gratton, E. http://www.fluorescence-foundation.org/lectures/ genova2007/lecture6.pdf (accessed April 2009).

(49) Xu, C.; Webb, W. W. Measurement of two-photon excitation cross sections of molecular fluorophores with data from 690 to $1050 \mathrm{~nm}$. J. Opt. Soc. Am. B 1996, 13, 481-491.

JP903727N 